\title{
Pancreatic Acinar Cell Cystadenocarcinoma
}

National Cancer Institute

\section{Source}

National Cancer Institute. Pancreatic Acinar Cell Cystadenocarcinoma. NCI Thesaurus.

Code C5727.

A cystic adenocarcinoma characterized by the presence of relatively uniform neoplastic cells which produce pancreatic enzymes and are arranged in acinar patterns. Signs and symptoms include abdominal pain, weig ht loss, nausea, and diarrhea. It usually has an aggressive clinical course. 